\title{
Des initiatives locales européennes pour atteindre le facteur 4 ?
}

\section{Elsa Mor}

\section{(C) OpenEdition}

\section{Journals}

\section{Édition électronique}

URL : http://journals.openedition.org/developpementdurable/8759

DOI : 10.4000/developpementdurable.8759

ISSN : 1772-9971

Éditeur

Association DD\&T

\section{Référence électronique}

Elsa Mor, « Des initiatives locales européennes pour atteindre le facteur 4 ? », Développement durable et territoires [En ligne], Vol. 2, n 1 | Mars 2011, mis en ligne le 25 juillet 2014, consulté le 03 mai 2019. URL : http://journals.openedition.org/developpementdurable/8759; DOI : 10.4000/ developpementdurable.8759

Ce document a été généré automatiquement le 3 mai 2019.

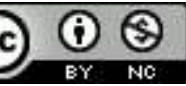

Développement Durable et Territoires est mis à disposition selon les termes de la licence Creative Commons Attribution - Pas d'Utilisation Commerciale 4.0 International. 


\title{
Des initiatives locales européennes pour atteindre le facteur 4 ?
}

\author{
Elsa Mor
}

1 Le changement climatique nous confronte à un enjeu mondial qui s'affranchit de toutes les frontières actuelles. À quelle échelle stratégique, des solutions peuvent-elles être apportées pour répondre à ce défi ? Après l'échec des négociations internationales pour le climat, à Copenhague, en décembre 2009, les espoirs fondés sur des leviers internationaux semblent compromis. Ne faudrait-il pas, dès lors, se tourner vers l'échelle locale, pour agir à la source des émissions de $\mathrm{CO}_{2}$ ? Une approche efficace dans la protection du climat ne réside-t-elle pas dans une approche multiscalaire, allant du local au global? Nous tenterons dans cet article de démontrer que l'action territoriale est un atout important dans le facteur 4, dont l'objectif est de diviser par quatre les émissions de gaz à effet de serre d'ici 2050.

Dans un contexte de mondialisation où les changements dans les politiques nationales et internationales sont diffus et lents, les pôles urbains peuvent contribuer à concrétiser, à brève échéance, des politiques efficaces et volontaires de réduction des émissions de gaz à effet de serre. Dans son ouvrage The renewable city Peter Droege (2006) précise que les phénomènes de globalisation engendrent des systèmes centralisés, ayant des rendements énergétiques faibles, faisant appel à des ressources énergétiques fossiles et nucléaires peu chères, qui façonnent, entre autres, des processus d'étalement urbain. À l'inverse, un retour vers le local aboutit à un processus de décentralisation des systèmes énergétiques - en faisant appel aux énergies renouvelables - à des stratégies de redensification et à une plus grande autonomie énergétique des pôles urbains. Comme le mentionne P. Calame (2009), un glissement s'opère et ce faisant, le territoire, longtemps perçu comme un résidu du passé, devient un acteur de l'avenir. Mais sera-t-il à même de répondre au défi du facteur 4 à l'horizon 2050, en se libérant progressivement des freins auxquels il est confronté ? Saura-t-il s'approprier les nouveaux paradigmes indispensables pour réussir une transition énergétique décarbonée, en faveur du climat? 
3 Tout d'abord, il s'agira d'interroger la focale locale que représentent le territoire et la ville, afin de cerner et de justifier la nécessité d'agir au niveau local en faveur du climat et de la sortie des énergies fossiles. Ensuite, nous examinerons les leviers et les freins des initiatives urbaines sectorielles, favorisant l'émergence d'un processus menant au facteur 4 à l'horizon 2050. Au-delà de l'approche sectorielle, nous étudierons les stratégies intégrées pour le climat, dont les plans énergie-climat. De plus, nous aborderons les difficultés liées aux conflits de temporalités, d'intérêts et les sentiers de dépendance, pour construire une gouvernance adaptée, propice au facteur 4.

\section{Quels sont les apports et les limites du cadre et du périmètre d'actions locales?}

4 De manière exponentielle, les pôles urbains se révèlent être à la fois d'importants consommateurs de combustibles fossiles et de grands émetteurs de gaz à effet de serre. Ils représentent, en effet, $1 \%$ de la surface terrestre, accueillent la moitié de la population mondiale et consomment $63 \%$ de la part d'énergie globale (WEO, 2008). À l'horizon 2030, plus de $60 \%$ de la population mondiale vivra en ville (Worldwatch Institute, 2007) et les villes seront responsables de $73 \%$ de la part d'énergie globale et de $76 \%$ des émissions de $\mathrm{CO}_{2}$ globale, selon le scénario de référence (WEO, 2008). Ces tendances suggèrent qu'il est impératif d'opérer au niveau local une transition énergétique pour répondre au facteur 4 à l'horizon 2050. Disposant d'un rayonnement au cœur de leur biorégion, les centres urbains possèdent un large panel de potentiels locaux, pour répondre aux défis globaux. Une multitude de territoires se mobilise pour réduire leurs besoins énergétiques et leurs émissions de $\mathrm{CO}_{2}$, sur le court, moyen et long terme.

Quelle définition et délimitation donnons-nous à une ville ou un territoire? Dans le World Energy Outlook de 2008, il est fait mention qu'il n'existe pas de consensus international sur la définition d'une ville et dans ce rapport, la ville correspond à une aire urbaine. La ville ou le territoire peuvent être considérés à la fois comme une entité vivante, un acteur institutionnel ou une entité géographique. Par ailleurs, les territoires locaux reflètent une multitude d'échelles institutionnelles et stratégiques. P. Calame aborde la question sous un nouveau jour : le «territoire acteur " rime trop souvent avec « collectivités territoriales », alors que le territoire est le lieu d'une pluralité d'acteurs, de systèmes, d'activités, de réseaux, et il ne peut donc être considéré seulement comme une institution politique (Calame, 2009). Selon P. Droege (2006), la ville est avant tout une entité socio-économique, culturelle et politique et ensuite, un système physique. En conséquence, en fonction de l'angle d'approche, les points de vue divergent.

6 La question du périmètre est centrale : comment les villes peuvent-elles délimiter leur périmètre d'action ? Sur quels postulats se fondent-elles ? Comme le WEO l'indique, il est délicat, à ce jour, d'opérer des comparaisons entre les villes, car d'une part, les frontières territoriales sont poreuses et d'autre part, il s'agit de bien mettre en rapport : ce que la ville consomme en matière d'énergie, comment elle est alimentée en énergie et ses parts d'émissions directes et indirectes. La connaissance des flux territoriaux énergétiques, des émissions et des matières premières, est donc cruciale et reste très complexe. À ce jour, les villes, dans leur ensemble, manquent de données chiffrées sur leurs consommations d'énergie et leurs émissions de GES. Elles n'ont également pas connaissance de l'ensemble des flux de marchandises, de matières premières et d'énergie qui les traversent. Les 
émissions indirectes sont, à ce jour, peu prises en compte. Comment les répartir et quelle part de ces émissions revient aux territoires producteurs de ressources manufacturières, à l'acheminement de marchandises et aux territoires consommateurs ? N'est-il pas biaisé de prendre en compte uniquement les émissions produites localement, alors que la part des émissions indirectes suppose vraisemblablement une part non négligeable des émissions globales?

7 Dans le cadre des plans climat énergie territoriaux, il est difficile d'évaluer et d'avancer efficacement sans intégrer et se réapproprier la connaissance des flux et du métabolisme urbain. Ce handicap rend délicate la réalisation d'un diagnostic d'origine (point zéro) qui permettrait ensuite aux territoires, d'évaluer leurs politiques énergie-climat. Par ailleurs, un grand nombre de villes recueillent leurs propres données de consommation d'énergie et d'émissions induites. Toutefois, les systèmes de reporting sont différents selon les territoires. En somme, un important travail d'homogénéisation doit être établi pour pouvoir comparer les territoires entre eux. À ce titre, ICLEI travaille actuellement à l'élaboration de méthodes de collecte de données plus homogènes (WEO, 2008). Selon P. Droege (2009), plusieurs raisons seraient à l'origine d'une relative efficacité des initiatives locales. La volonté de se focaliser sur des stratégies de quantification des émissions et des objectifs de réduction des émissions de GES, peut s'avérer être une stratégie biaisée, consistant à retarder l'échéance de l'action. Ainsi, toujours d'après lui, il serait plus judicieux de mettre en place, dès maintenant, les leviers d'action les plus efficaces, pour atteindre un contenu "zéro combustible fossile", en matières de consommation d'énergie et d'énergie grise ${ }^{1}$, dans tous les secteurs d'activité. De plus, il dénonce le fait que depuis bientôt 20 ans d'efforts et de concertations, aucun accord, même de principe, n'ait pu émerger en matière de méthodes de quantification des émissions (alors que le protocole de Kyoto fournit un cadre aux quantifications nationales relatives aux émissions générées localement). Les émissions indirectes n’y étant pas intégrées, l'évaluation reste imprécise.

8 Au-delà de ces freins et de ces critiques, auxquels il est crucial d'apporter des réponses efficientes, les villes peuvent, en parallèle, mettre en place des dispositifs énergie-climat efficaces, s'appuyant sur les leviers directs ou indirects. La complexité du défi repose sur la capacité à les combiner sur le court et le long terme, pour s'inscrire dans une trajectoire post-carbone, et atteindre le facteur 4 à l'horizon 2050. Ces perspectives supposent un changement de paradigme énergétique, afin de distiller et de planifier, dès aujourd'hui, cet objectif dans l'ensemble des politiques et des actions locales menées à court, moyen et long termes. Dès lors, la hiérarchisation des priorités constitue un préalable à l'action.

9 Selon le maire de Malmö, Ilmar Reepalu, les municipalités doivent jouer un rôle de pionnier en termes d'efforts pour la protection du climat. Selon l'organisation allemande "Climate Alliance », en 2009, elles ont un potentiel moyen de $10 \%$ de réduction des émissions de GES du territoire. Décliné, ce potentiel permet aux municipalités de réduire leurs consommations d'énergie et leurs émissions dans leurs patrimoines immobiliers de l'ordre $25 \%$ à $60 \%$ pour le chauffage et de plus de $10 \%$ pour l'électricité. La réhabilitation des bâtiments existants peut aller jusqu'à une réduction de $20 \%$ des émissions totales de $\mathrm{CO}_{2}$. Les comportements des utilisateurs et les petits investissements contribuent pour environ $5 \%$. En matière de transport, les municipalités, à travers leur parc de véhicules et leurs compétences en matière d'urbanisme et d'aménagement peuvent réduire de $30 \%$ leurs émissions. Leur exemplarité les rend crédibles et contribue à gagner la confiance 
des acteurs du territoire et des citoyens et à renforcer leur implication dans cette dynamique. En tant qu'actrices de la planification locale, elles influent à la fois sur la localisation, le développement de nouveaux projets urbains; l'application de référentiels performants de construction ; l'optimisation des politiques de mobilité ; la gestion, le tri et le recyclage des déchets; ainsi que sur les réseaux de distribution de l'énergie via leur pouvoir de concession. Elles peuvent également, au travers de leurs outils de communication, sensibiliser et conseiller les citoyens et les acteurs du territoire. En somme, à l'échelle d'un territoire, la part des émissions de $\mathrm{CO}_{2}$ d'une collectivité reste négligeable, mais son rayonnement local peut lui permettre d'impulser des dynamiques territoriales.

\section{Leviers et freins des gisements potentiels de réduction des consommations d'énergie fossiles et des émissions de $\mathrm{CO}_{2}$}

10 Il est généralement reconnu que les principaux secteurs d'émissions sont les transports et le bâtiment. Cependant, les gisements de réduction des émissions de $\mathrm{CO}_{2}$ se trouvent également dans les infrastructures et les systèmes de production énergétiques locaux et les politiques d'urbanisme.

\subsection{La course aux standards énergétiques dans le bâti : gains substantiels pour réduire l'empreinte carbone du territoire}

11 De nombreuses villes développent des plans d'action en direction du secteur du bâtiment, avec d'importants chantiers de rénovation du patrimoine bâti existant, et la mise en place de codes de construction et de standards énergétiques. C'est le cas, par exemple, de Frankfort et Freiburg, qui ont créé des référentiels plus ambitieux que ceux mis en place au niveau national. Cela permet d'évaluer la part des émissions potentielles qui seront évitées dans les prochaines décennies. Dans le cas de Barcelone, l'ordonnance solaire est un levier innovant d'efficience énergétique dans le domaine de la production d'électricité et de chaleur renouvelable à l'échelle du bâtiment. Cette loi impose de produire à hauteur de $60 \%$ de l'eau chaude fournie par des systèmes solaires thermiques. Cette approche a été par la suite adoptée par le code national de construction d'Espagne (WEO, 2008).

Sachant que la durée de vie d'un bâtiment s'étend sur plus de 50 ans, la municipalité de Freiburg a décidé d'intégrer, dès la phase de conception des constructions de bâtiments, les composantes énergétiques, afin d'en optimiser les consommations. Dans les clauses municipales d'urbanisme, les constructeurs sont dans l'obligation d'intégrer des critères énergétiques ambitieux, dans la mesure où les coûts ne sont que de $10 \%$ plus élevés que la variante standard. Depuis 2009, les standards d'efficacité énergétique (chauffage : 15 $\mathrm{kWh} / \mathrm{m}^{2} / \mathrm{an}$ ) doivent s'appliquer au parc résidentiel de la municipalité. Depuis 2008, la ville a décidé que son patrimoine bâti et les nouveaux projets d'urbanisme de l'entreprise municipale de construction devaient être construits selon les normes passives (Climate Alliance). Depuis 1983, à Frankfort, à partir de réformes administratives et budgétaires, le service municipal de gestion de l'énergie, responsable de plus de 1800 équipements a réussi à économiser des coûts énergétiques de l'ordre de 400000 euros (Climat Alliance). Redistribués dans le domaine de l'énergie, cela lui permet de garantir une prévision de 
réduction de $24 \%$ des émissions de $\mathrm{CO}_{2}$ de ses équipements (par rapport à 1990) (Climat Alliance). De plus, à la faveur d'une résolution du conseil municipal, les nouvelles constructions de bâtiments municipaux, et celles relatives à des partenariats ou des rénovations doivent intégrer les normes passives. Ainsi, Frankfort devient la première ville à standardiser, en passif, la construction de bâtiments municipaux (Wuppertal Institute, 2009).

Ces exemples représentent des pratiques innovantes, mais qu'en est-il du parc existant privé ? À ce jour, il est encore délicat de réaliser vastes opérations de rénovation en direction du parc privé et les leviers mis en œuvre en la matière ne sont que très peu déployés pour inciter les propriétaires privés à rénover leurs biens immobiliers. L'un des freins au développement d'opérations éco-performantes en matière de construction neuve, de rénovation du patrimoine existant ou d'aménagement urbain, est bien résumé par le concept du « circle of blame » (ou cercle du reproche). Le déroulement-type en est le suivant: les constructeurs sont en capacité de construire des bâtiments écoperformants, mais ils ne veulent pas, car les promoteurs ne leur passent pas de commandes. Si les promoteurs ne leur passent pas de commandes, c'est parce que les investisseurs ne l'exigent pas, et ne seront pas enclins à financer ce type de projets. Les investisseurs ne l'exigent pas, car il y a une faible demande de la part des occupants. Enfin, les occupants ne leur demandent pas, car ils considèrent qu'il n'y a qu'un faible taux de logements de ce type disponibles (Cadman, 2007). La répartition des coûts de développement entre l'utilisateur final, les autorités locales, le promoteur et l'investisseur limite souvent des opérations éco-performantes de construction ou de rénovation de bâtiments, pour des raisons de rentabilité à court terme (Meijer et al., 2009).

Il semble important de dépasser ces liens de causalités, afin de mener des actions, dès maintenant, menant sur la voie du facteur 4 à l'horizon 2050, dans une perspective de backcasting (prospective du présent) en intégrant les objectifs ambitieux pour le parc existant et les normes passives ou positives pour le parc neuf. Cependant, les réflexes et la facilité aboutissent à l'installation de systèmes peu efficaces qui, à terme, nous en écartent. Ce processus de backcasting devrait être décliné à l'ensemble des secteurs d'activité et pour chaque projet.

\section{2. De multiples leviers dans le domaine du transport, rarement généralisés et combinés}

15 Dans le domaine du transport, des initiatives se sont déployées, pour réduire les phénomènes de congestion, de déplacements en véhicules individuels et promouvoir les modes de transports publics et la mobilité douce. Dans la ville de Graz, en Autriche, la dégradation de la qualité de l'air a incité la municipalité à généraliser une limitation de vitesse à $30 \mathrm{~km} / \mathrm{h}$ pour les véhicules à l'ensemble du territoire (hormis les grands axes). Les axes piétons et de vélo se sont élargis. Graz devient la première ville autrichienne à créer un centre de la mobilité et à déployer une nouvelle flotte de bus alimentée au carburant bio-diesel (H. Rohracher, P. Späth, 2009). Les territoires pilotes tels que les villes suédoises sont des niches d'expérimentation et d'innovations. En effet, de nombreuses municipalités convertissent leurs flottes de véhicules au biodiesel, produit localement ou incitent financièrement les habitants à acheter des véhicules alimentés au biodiesel. 
16 À Londres et à Stockholm, par exemple, le choix d'un péage urbain a constitué un engagement politique fort et un levier financier qui permet, à la fois, de financer des projets de transports publics qui, à moyen et long termes, réduiront les émissions de $\mathrm{CO}_{2}$. Depuis 2003, la ville de Londres a mis en place un péage urbain dans le centre ville. L'ensemble des bénéfices permet de financer les transports publics (lignes de bus supplémentaires). La capacité de transport ferroviaire public a augmenté avec l'ajout de 1200 wagons supplémentaires. Selon la compagnie londonienne d'exploitation, le péage urbain a permis de réduire le volume de circulation de $18 \%$. La perte de temps dans les embouteillages s'est réduite de $30 \%$, ce qui équivaut à une réduction d'environ 150000 tonnes de $\mathrm{CO}_{2}$ par an (Wuppertal Institute). Dans le cas de Stockholm, un péage urbain a été mis en place en 2007. Depuis 2005, la circulation en direction et à l'intérieur du centre ville a diminué de $18 \%$ environ et les phénomènes de congestion ont diminué de 30 à $50 \%$ selon les évaluations. Ainsi les émissions de gaz à effet de serre ont baissé à la faveur de la mise en place de ce péage urbain, dans le centre ville mais aussi, dans une moindre mesure, à l'échelle régionale (SALAR, 2009).

Ces projets réduisent la place de la voiture en ville et révèlent un certain courage de la part des acteurs politiques du territoire. En effet, l'action en direction des transports remet en cause les habitudes des habitants et engendre des contraintes en direction des administrés. Dès lors, les élus politiques, dans un souci de préserver leur électorat, préfèrent s'en détourner. Cependant, dans la majorité des situations, les autorités locales rencontrent des difficultés pour réguler et influer sur le trafic routier dans les principales artères et sur les performances environnementales des véhicules (Borgstede et al., 2007). En revanche, l'ensemble de ces initiatives, certes encore minoritaires, tendent à prouver qu'il est possible de réguler les déplacements, en privilégiant des modes doux, des systèmes incitatifs ou contraignants. Une combinaison de l'ensemble de ces initiatives donnerait un nouveau visage aux pôles urbains.

\subsection{Des leviers multifonctions d'aménagement urbain amenant à une réduction de la dépendance automobile et des émissions de $\mathrm{CO}$}

2

Les initiatives d'aménagement du territoire urbain et des projets de densification autour des axes de transports publics peuvent contribuer à créer des zones de faibles émissions. Dans cette perspective, l'agglomération grenobloise a adopté une charte urbanismetransport, intégrée au Plan de Déplacement Urbain. Un contrat d'axe est décliné pour chaque nouvelle ligne de tramway. L'intérêt est de planifier et de coordonner une densification au voisinage des nouvelles lignes. Il devient envisageable de voir se dessiner progressivement des territoires "bas-carbone» (Maür, 2009). Ce type d'opération contribue à décentrer la voiture des projets urbains et à proposer des alternatives pérennes sur le moyen et le long termes. Cependant, ces initiatives ne sont pas à l'abri d'effets rebonds (hausse de circulation de véhicules individuels). Il est ainsi important que ces stratégies d'aménagement soient accompagnées de campagnes de sensibilisation, afin que les habitants se détachent progressivement de leur véhicule motorisé et choisissent les transports collectifs.

L'aménagement urbain peut également renforcer une diminution des consommations d'énergie et limiter les déperditions énergétiques, via une économie d'échelle. En effet, les 
schémas de développement territorial et de planification peuvent se focaliser sur la réalisation d'opérations autour d'un axe de tramway, mais également autour des axes d'un réseau de chaleur, afin d'en optimiser l'utilisation. La combinaison des problématiques d'aménagement urbain et d'infrastructure énergétique est illustrée dans l'exemple de l'Oosterdok Island, à Amsterdam. Au cœur de ce projet, les investisseurs, les promoteurs et les résidents sont les copropriétaires du système énergétique qui alimente le quartier. Ainsi la fourniture d'énergie et la maintenance sont garanties, à long terme, et les parties prenantes bénéficient de retours et d'un faible prix de l'énergie. La réduction des émissions de $\mathrm{CO}_{2}$ de ce système est d'environ 64\% (Meijer et al., 2009).

\subsection{La reprise en main de la production d'énergie}

Dans le contexte actuel, un intérêt accru des collectivités territoriales, pour disposer d'une compétence énergie fait émerger des initiatives innovantes, notamment par la création d'entreprises locales d'énergie.

Londres, à travers son plan climat-énergie, développe des projets en partenariat avec des entreprises privées, pour réaliser et financer des opérations « zéro carbone » (WEO, 2008). La consommation de gaz et d'électricité de la ville émet 35 millions de tonnes de $\mathrm{CO}_{2}$ par an, soit $75 \%$ des émissions totales. Moins de $10 \%$ d'électricité et environ $5 \%$ de chaleur sont produites localement. Le Maire privilégie la production locale décentralisée d'énergies faiblement carbonées. Son objectif vise à produire localement un quart des besoins énergétiques du territoire, d'ici à 2025, et la moitié d'ici à 2050, à partir de centrales de cycles combinés gaz et des sources d'énergie renouvelables. À cet effet, il a constitué The London Climate Change Agency (LCCA). En 2006, en partenariat avec EDF Énergie, la LCCA a mis en place une entreprise municipale de services énergétiques : ESCo Londres, par le biais d'une joint-venture. Fruit d'un partenariat expérimental public/ privé, la coopération multi-acteurs révèle des mécanismes de gouvernance innovants. ESCo Londres a la charge de concevoir, financer, construire et gérer l'exploitation des réseaux d'énergie décentralisés locaux. Les projets en cours seraient à même de concrétiser $8 \%$ du potentiel de cogénération de la ville (GLA, 2007). Malgré ces initiatives innovantes et prometteuses, la municipalité de Londres et le Grand Londres peuvent contribuer à réduire de seulement $15 \%$ les émissions du territoire. Afin de tenir l'objectif de réduction de $60 \%$ des émissions du territoire d'ici 2025 , la responsabilité doit impérativement être partagée avec l'ensemble des acteurs du territoire, dont les entreprises, les institutions publiques (35-40\%), les habitants (5-10\%) et l'État (30\%) (Hammer, 2009).

\subsection{Des exemples d'initiatives performantes de transition énergétique}

Dans le cas de Copenhague, de Graz, de Vaxjö, de Stockholm, la transition énergétique s'est portée sur la diversification du mix énergétique de leurs réseaux de chaleur, notamment en privilégiant les biocombustibles au détriment des combustibles fossiles.

La ville de Copenhague détient l'un des réseaux de chaleur les plus importants du monde. Avec une infrastructure de $1300 \mathrm{~km}$, il alimente plus de 70\% des logements et d'autres zones urbaines. Depuis 1984, le système est supervisé par deux entreprises en partenariat avec la ville. Plus des $2 / 3$ de l'énergie provient d'un système de cogénération et est 
alimenté principalement par le gaz naturel et des biocombustibles. Dans le milieu des années 1990, selon les données de la municipalité, le changement de combustible (du charbon par le gaz puis par les biocombustibles) contribue à réduire les émissions de $665000 \mathrm{tCO}_{2} / \mathrm{an}$. Le succès du réseau de chaleur est en partie dû à un allègement fiscal introduit dans les années 1980, à l'échelle nationale en direction des centrales de cogénération. Ceci contribue à fournir une chaleur à bas prix (Wuppertal Institute, 2009). La ville de Vaxjö a entamé, à la fin des années 1990, un chantier ambitieux de sortie des énergies fossiles, qui se décline dans l'ensemble des secteurs (habitat, transport, énergie...). L'objectif fixé à l'échelle de la ville est de réduire de moitié les émissions de gaz à effet de serre d'ici 2010. La conversion aux biocombustibles et les systèmes de cogénération des réseaux de chaleur participent, pour une grande part, à la réduction des émissions de $\mathrm{CO}_{2}$ dans le domaine de l'énergie. Les subventions accordées aux habitants pour se raccorder au réseau de chaleur représentent un levier supplémentaire. Ainsi, plus de $76 \%$ des logements sont alimentés par le réseau de chaleur, aboutissant à une division par 4 des émissions de $\mathrm{CO}_{2}$ dues au chauffage. Les émissions totales de $\mathrm{CO}_{2}$, qui étaient de 4,6 t/hab. en 1993, sont réduites de $32 \%$, soit 3,12 t/hab. en 2007 (Emelianoff, 2009).

Dans une perspective de neutralité carbone, d'autonomie énergétique et de relocalisation de la production, une politique de transition énergétique, orientée vers les énergies renouvelables, contribue à détacher les territoires à la fois de leur dépendance aux réseaux nationaux et aux énergies fossiles. La création d'emplois, le développement de nouvelles technologies, une utilisation responsable des ressources locales (Droege, 2006) et de nouveaux modèles de gouvernance représentent les pendants positifs de ce processus. En revanche, les effets pervers du développement des réseaux de chaleur révèlent un niveau de dépendance élevé aux déchets, qui n'encourage pas l'introduction de sources d'énergies renouvelables.

Dans un autre registre, des nouveaux systèmes de coopération multi-acteurs voient le jour, comme à Copenhague. En l'an 2000, un groupe, comprenant des citoyens danois, les autorités locales, des entreprises et des associations, s'est réuni pour constituer une coopérative. Ils sont à l'initiative de la construction d'un parc offshore de 40 éoliennes. Ce parc produit environ 90 millions de kWh d'électricité, équivalent à $3 \%$ de l'électricité consommée à Copenhague. Les éoliennes économisent plus de $76000 \mathrm{tCO}_{2}$, chaque année et fournissent 40000 logements en électricité. Plus de 8500 individus et quelques organismes ont contribué à financer le projet de coopérative (Wuppertal Institute, 2009). Ainsi, la diversité des parties prenantes, avec l'implication des habitants, des entreprises et des autorités locales permet d'initier des politiques ambitieuses en matière d'énergies renouvelables et de réduction des émissions de $\mathrm{CO}_{2}$. Dans la filière éolienne, cependant, il existe un certain nombre de freins au développement. Les investissements s'orientent davantage vers l'amélioration de l'efficacité énergétique et de l'intensification de l'utilisation des systèmes de production existants, au détriment des nouveaux, issus des énergies renouvelables (Söderholm et al., 2007). Dans de nombreux territoires européens, la résistance du public représente un obstacle à l'essor de parcs éoliens. Des études menées en Suède concluent que les freins locaux liés au manque de portage politique, à la réticence du public et à la faible implication des acteurs locaux peuvent être à l'origine de la non-atteinte des objectifs nationaux (Söderholm et al., 2007). La durée du processus d'obtention des permis de construire peut s'étaler sur plus de 7 ans et représente ainsi un obstacle au développement des renouvelables. Le cas de l'éolien illustre les obstacles que rencontrent les innovations et les nouvelles technologies de l'énergie. Elles subissent une 
forme de "paternalisme conservateur ", conséquence d'une "dépendance au sentier " vis-à-vis des systèmes énergétiques en place.

Enfin, la diversité et la multiplicité des initiatives locales peuvent aboutir à une fragilisation, provoquée par leur fragmentation, leur manque de cohérence et de coordination. Ce talon d'Achille ne facilite pas l'évaluation des actions développées. L'émergence des plans climat énergie territoriaux serait susceptible de donner une plus grande cohésion aux initiatives locales. En Suède, la volonté d'une politique climatique nationale déclinée à l'échelle locale a permis une réduction de plus de $20 \%$ des émissions nationales, au cours des 20 dernières années (Emelianoff, 2009).

\section{L'apport des Plans Climat Énergie Territoriaux}

L'un des outils intéressants dont dispose une collectivité territoriale est le Plan Énergie Climat Territorial (PECT). Il consiste à déployer une stratégie locale, susceptible d'être déclinée à chaque échelon territorial. Il s'agit, d'abord, de réaliser un diagnostic territorial des sources d'émissions de GES et d'identifier leurs évolutions. Ensuite, un plan d'action est défini afin de réduire à la source les émissions de GES, de mobiliser l'ensemble des acteurs du territoire et d'atténuer les vulnérabilités territoriales, par des mesures d'adaptation. Cette démarche se concrétise par l'instauration d'un calendrier, d'objectifs, d'indicateurs quantitatifs et qualitatifs, d'un plan d'action et de scénarios à moyen et à long terme. Elle permet d'esquisser des visions du futur, contribuant à répondre au facteur 4 d'ici 2050, afin d'identifier les leviers pour opérer une mutation et une transition énergétique. À travers cet outil, l'évaluation des secteurs ou des infrastructures émetteurs de $\mathrm{CO}_{2}$ peut conduire à hiérarchiser les priorités d'action, pour réduire l'empreinte carbone des territoires.

Dans les années 1990, certaines villes suédoises comme Stockholm, Malmö ou Vaxjö se sont fixé des objectifs très ambitieux de réduction des émissions de $\mathrm{CO}_{2}$. C'est notamment le cas de Vaxjö avec une politique innovante et efficace, visant à diviser par deux ses émissions, à l'horizon 2010. À travers son plan d'action, initié en 2007, la ville de Londres s'inscrit également dans une trajectoire audacieuse, en se fixant un objectif de $60 \%$ de réduction des émissions de son territoire, à l'horizon 2025 (par rapport à l'année 1990). Dans le cas de Graz, en Autriche, le Plan Climat a émergé au début des années 1990, à l'initiative du nouveau responsable du pôle environnement. L'objectif était de réduire de $50 \%$ les émissions de gaz à effet de serre d'ici 2010, en référence aux émissions de l'année 1987 (Rohracher, Späth, 2009). Dans ce dernier exemple, la gouvernance s'avère être un atout de taille. En effet, les politiques climat-énergie s'intègrent au cœur de l'ensemble des politiques sectorielles. En matière d'urbanisme, les initiatives ont été développées par des acteurs extérieurs (instituts de recherche), en relation étroite avec les services de la municipalité, ce qui participe à une plus grande légitimité des recommandations proposées. Enfin, l'élaboration du plan d'action a été un exercice collectif regroupant une multitude d'acteurs (associations, entreprises, équipes de recherche, services municipaux), se répartissant les actions à conduire pour réduire les émissions territoriales de $\mathrm{CO}_{2}$. Il s'en est suivi une mobilisation des citoyens (Rohracher, Späth, 2009).

Outil expérimental, le plan Climat est propice à la valorisation de nouveaux systèmes de gestion et d'organisation, supposant une transversalité, une intégration des politiques 
publiques et des partenariats. En effet, le PCET introduit de nouveaux critères dans le choix des politiques publiques, il apporte un argumentaire commun sur les différents projets mis en œuvre et vise une coordination et une meilleure cohérence des politiques. Si l'objectif est l'intégration d'une dimension climat au cœur de l'ensemble des politiques publiques, le plan Climat se heurte comme toute démarche transversale aux prérogatives des différents services et niveaux administratifs. Il peut être délicat à terme d'évaluer les segments de ce dispositif, et de faire la part entre les évolutions spontanées des émissions et celles qui résultent d'arbitrages politiques. De plus, il ne s'inscrit pas dans la perspective de déployer une réelle politique spécifique de prévention contre les chocs pétroliers. En réalité, l'intérêt accru pour la problématique climatique estompe les enjeux liés à la raréfaction des ressources fossiles. En effet, à ce jour, au niveau international, nous avons identifié un certain nombre de territoires ayant signé une résolution au regard du pic pétrolier. Cependant peu nombreux sont encore les territoires disposant d'un plan d'action détaillé de réduction de l'utilisation du pétrole, s'inscrivant dans une trajectoire de sortie des énergies fossiles (Newman et al., 2009). En Amérique du Nord, quelques villes comme Portland et San Francisco, notamment, ont développé des Peak Oil Task Force et des politiques énergétiques, pour intégrer cette question au cœur des stratégies locales.

\section{Comment les conflits de temporalités, les divergences d'intérêts et les dépendances au sentier viennent ralentir l'émergence d'une gouvernance efficace en faveur d'initiatives locales?} temporalité contribuent à freiner l'action des multiples organisations en présence. En d'autres termes, n'évoluant pas au même rythme, la capacité d'action de certains acteurs peut être gênée par d'autres, évoluant dans des sphères temporelles plus longues. Ainsi, la dynamique de projet est biaisée, s'étire dans le temps et perd de son efficacité. Sachant que les processus de changement urbain sont longs et sujets à de fortes inerties, il semble impératif que les acteurs du territoire combinent les échelles temporelles en présence, pour identifier les priorités d'action.

Viennent s'ajouter les conflits d'intérêts. En effet, du point de vue institutionnel, les instances territoriales existantes, confrontées aux défis énergétiques et climatiques, ont comme alternative : soit de définir une approche sectorielle, dans un univers hiérarchisé, soit d'intégrer une approche spécifique climat - énergie, dans un univers multi-acteurs, multi-niveaux et interinstitutionnel. Ces défis, s'affranchissant des frontières administratives, organisationnelles, politiques et sectorielles, accentuent l'interdépendance de ces sphères. Cela provoque un dilemme social, chez les décideurs, en matière d'ouverture, de coopération et de partenariat notamment. Au sein des administrations ou des entreprises, ils doivent évaluer et questionner la légitimité d'une politique climatique, susceptible d'être en conflit avec des valeurs, des intérêts et des normes organisationnelles et professionnelles profondément ancrés (Lundqvist, Biel, 2008). D'après L. J. Lundqvist et A. Biel (2008), les décideurs, à différents niveaux et dans différentes sphères sectorielles, sont prisonniers de réglementations et de procédures, qui limitent leurs marges de manœuvre, pour se réorganiser et se réorienter vers de 
nouvelles activités, davantage respectueuses de l'environnement. En d'autres termes, le changement climatique défie les visées souvent conservatrices des acteurs publics et privés. Il suppose, en effet, une refonte interne importante de l'ensemble des systèmes organisationnels, qui semble, à ce jour, encore délicat à réaliser.

De plus, selon P. Droege (2006), les responsables locaux, chargés de superviser des processus de prise de décision collective, sont davantage formés à administrer, sur un mode réactif que d'anticiper ou planifier en amont. Ainsi, la capacité de surmonter ce défi institutionnel local s'inscrit au cœur d'un enjeu plus global : les villes seront-elles à même d'anticiper ou deviendront-elles davantage vulnérables, devant les phénomènes d'accélération des conséquences du changement climatique et les répercussions du pic pétrolier? Les défis énergétiques et climatiques ne supposent pas nécessairement de nouvelles institutions territoriales. Cependant, elles exigent de nouveaux modèles de gouvernance, de prise de décision, d'engagement et de responsabilité, pour mener à bien des projets efficients, dans le but de s'inscrire sereinement dans une trajectoire de facteur 4 d'ici 2050 (Droege, 2006).

Les mécanismes de gouvernance doivent être structurés de manière robuste, afin de démêler les complexités des réseaux de politique locale, en intégrant la fragmentation des institutions et les cloisonnements entre les modes de fonctionnement différents des acteurs locaux et les valeurs opposées qui les portent. Le néo-institutionnalisme, à travers ces trois écoles de pensée (l'institutionnalisme historique, des choix rationnels et sociologique), renouvelle l'analyse institutionnelle. Il peut potentiellement constituer une approche appropriée, pour disséquer les systèmes de gouvernance énergétique et climatique urbaine, notamment en examinant les sentiers de dépendance institutionnelle (Hammer, 2009). Ces écoles cherchent toutes à élucider le rôle joué par les institutions dans la détermination des résultats sociaux et politiques (Hall et al.,1997). L'institutionnalisme historique, s'intéressant à la compréhension des réformes, met l'accent sur les dépendances au sentier, à travers l'idée selon laquelle les institutions héritées du passé continuent à produire leurs effets par-delà la réforme (Aust, 2003). Ils se traduisent par les héritages et le poids des habitudes qui rendent difficiles les bifurcations dans une perspective de sortie des énergies fossiles et de décarbonisation des territoires.

\section{L'articulation des échelles territoriales dans les processus de gouvernance, facteur contraignant ou vecteur d'opportunités?}

La multiplicité des échelles territoriales vient ajouter une dimension supplémentaire à la complexité des enjeux et des stratégies. Dans le cas de la France, chaque échelle institutionnelle est, en effet, libre d'établir une politique énergie-climat. Ainsi, le questionnement porte davantage sur la gouvernance territoriale, et l'interaction entre les échelles et les acteurs que sur les compétences et les périmètres administratifs. Comme l'explique P. Calame, ne faut-il pas reconstruire une pensée sur la gouvernance ? L'enjeu en effet est d'éviter de centrer cette dernière uniquement sur le partage des compétences, au profit d'une l'articulation des actions des différentes collectivités, présentes sur un territoire donné. Ce glissement permettrait d'élaborer une compétence partagée, sur les bases du principe de subsidiarité active et non un simple partage des compétences. En d'autres termes, la création de compétences énergie-climat partagées, 
interinstitutionnelles, éviterait un empilement d'initiatives et de plans fragmentés et donnerait ainsi davantage de cohérence au projet de facteur 4 à l'horizon 2050, à une échelle territoriale donnée.

Se pose alors la question de l'articulation des échelles institutionnelles. Dans le contexte actuel, l'échelle locale semble être la plus stratégique pour déployer des actions concrètes de réduction à la source des émissions de $\mathrm{CO}_{2}$. Dans une perspective plus générale, la solution ne réside-t-elle pas dans la dynamique produite par une articulation des échelles territoriales? Les systèmes de gouvernance multi-niveaux, auxquels participent les villes peuvent être appréhendés comme un facteur contraignant, autant qu'un vecteur d'opportunités. Ce peut être une contrainte, compte tenu de l'existence de coûts de coopération. Mais c'est une source d'opportunité en raison de possibles économies d'échelle et de bénéfices sociaux élargis du fait précisément de la coopération. Des initiatives territoriales peuvent ainsi favoriser le changement, à l'échelle régionale et nationale. Les cas de Freiburg et Barcelone sont des exemples qui ont permis de légitimer des réformes, dans le domaine de l'énergie à l'échelle du bâtiment au niveau national. Les leviers locaux combinés à des politiques régionales et nationales, peuvent donc contribuer efficacement au facteur 4, à condition qu'elles soient coordonnées et valorisées.

En revanche, les municipalités peuvent parfois être confrontées à des situations délicates, prises en tenaille par des projets d'aménagement, votés et amendés par des échelons institutionnels supérieurs (voie ferroviaire, autoroute, aéroport, réseaux haute tension). Elles n'ont alors que peu d'emprise et de marges de manœuvre à leur actif. Cela nuit à l'émergence d'un consensus local, ou rend contre-productives les politiques énergieclimat conduites (effets rebond engendrés par l'aménagement de nouvelles infrastructures routières). Il semblerait pertinent, pour analyser et cerner au mieux les réponses et les actions sur le plan local, d'intégrer les articulations d'échelles territoriales et les aspects organisationnels et de gouvernance, pour opérer une transition climatique et énergétique efficace.

\section{Conclusion}

Tout au long de l'article, il est apparu que les vecteurs de réussite d'une action locale efficace, en faveur du climat et de la résilience énergétique des territoires, se caractérisent par un portage politique fort, un intense effort de sensibilisation, des budgets élevés, affectés aux projets innovants, des incitations économiques en faveur de l'efficacité énergétique, des coopérations multi-acteurs. Il en va de même des politiques combinées, fédérant un grand nombre d'acteurs locaux. Cependant, de nombreux freins persistent et se doivent d'être davantage étudiés. Dans le secteur du bâtiment, la rénovation de l'existant ou en direction du patrimoine privé reste plus complexe et coûteuse que dans la construction neuve. La formation des filières et des corps de métier aux techniques innovantes semble essentielle. La résistance au changement est une difficulté majeure, qui se décline dans l'ensemble des sphères d'action. Les opérations de densification autour des axes de transport collectif ne sont pas à l'abri des effets rebonds. Le lobbying des grands opérateurs et les coûts élevés d'investissement dans la construction de nouvelles infrastructures énergétiques locales peuvent freiner les initiatives. Les innovations technologiques et énergétiques, dans le domaine des énergies renouvelables, s'imposent difficilement. 
ation des échelles territoriales est un levier prépondérant, pouvant bénéficier des effets d'échelle et doit également se décliner entre les différentes sphères scientifiques, sociales, économiques, juridiques, philosophiques, qui caractérisent les sociétés actuelles. Ce n'est qu'avec cette "conception intégrée », que pourrait se construire un langage commun et une conscience collective, à même d'initier une transition harmonieuse vers le facteur 4. Les villes s'avèrent constituer le bon niveau d'approche pour la résolution des enjeux climatiques et énergétiques. Des travaux de recherche centrés sur le métabolisme urbain, la quantification des émissions de GES et sur les signaux et les facteurs déclencheurs propices au changement, à l'échelle urbaine, favoriseraient davantage l'efficacité des initiatives locales.

Enfin, les défis énergétiques et climatiques demandent aux villes, considérées comme « les graines du changement " (Droege, 2006), d'identifier les racines des futurs possibles et de construire des visions d'avenir. La dimension rétrospective nous rappelle que les villes sont les constructions les plus avancées, mais aussi les plus fragiles et périlleuses, jamais conçues par l'humanité (Newman et al., 2009). Il est donc essentiel de resituer la ville au cœur de sa biorégion, tant d'un point de vue énergétique, qu'agroalimentaire. Ce positionnement permet à la fois de concrétiser le facteur 4, favorisant l'émergence de territoires post-carbone (Theys, 2009) et d'optimiser la résilience des territoires. C'est en évoquant la multitude des initiatives locales à l'œuvre dans le monde, qu'Edgar Morin (2010) affirme: «ce sont ces voies qui pourront, en se développant conjointement, se conjuguer pour former la voie nouvelle, laquelle nous mènerait vers l'encore invisible et inconcevable métamorphose».

\section{BIBLIOGRAPHIE}

AIE, 2008, World Energy Outlook 2008, IEA Book, France

Cadman D., 2007, Centre for the Study of Sustainable Building: The Carbon Challenge, Londres, http://www.bne.uwe.ac.uk/cssb/docs/carbonchallenge_davidcadman.pdf

Borgstede C. von, Zannakis M., Lundqvist L. J., 2007, "Organizational culture, professional norms and local implementation of national climate policy", in Lundgvist L. J., Biel A., From Kyoto to the Town Hall : Making International and National Climate Policy Work at the Local Level, Earthscan Publications, London, p. 77-92

Calame P., 2009, Territoire : des mots pour le dire, Exposé aux Assises nationales « Énergie et climat » des collectivités locales en France, Conférence, 28 janvier

Droege P., 2006, The renewable city, A Comprehensive Guide to an Urban Revolution, John Wiley \& Sons Ltd, Royaume-Uni

Droege P., 2009, 100\% Renewable, energy autonomy in action, Earthscan Éditions

Emelianoff C., 2009, Stratégies locales de sortie des énergies fossiles : exemple de 4 villes européennes,

Växjö, Stockholm, Fribourg et Hanovre, Liaison Énergie Francophonie

Développement durable et territoires, Vol. 2, $\mathrm{n}^{\circ} 1$ | Mars 2011 
GLA, 2007, Action Today to Protect Tomorrow, The Mayor's Climate Change Action Plan, Greater London Authority, Londres

Hall P. A., Rosemary C. R., 1997, « La science politique et les trois néo-institutionnalismes », Revue française de science politique, Volume 47, $\mathrm{n}^{\circ}$ 3, p. 469-496

Hammer S. A., 2009, Capacity to act : The critical determinant of local energy planning and program implementation, Columbia University, Actes du Symposium : les Villes et le changement climatique, Banque Mondiale, Marseille

Lundgvist L. J., Biel A., 2007, From Kyoto to the Town Hall : Making International and National Climate Policy Work at the Local Level, Earthscan Publications, London

Maür A.-M., 2009, Comment canaliser l'urbanisation à long terme autour d'infrastructures de transports publics ?, Exposé au quatrième séminaire du programme «Ville Post-Carbone », 13 novembre

Meijer M., Adriaens F., Linden O., Schik W., 2009, The Next Step for sustainable urban design in the Netherlands', 45th ISOCARP Congress

Morin E., 2010, « Éloge de la métamorphose », Le Monde, 10 et 11 janvier

Newman P., Beatley T., Boyer H., 2009, Resilient Cities: Responding to Peak Oil and Climate Change, Island Press

Rohracher H., Späth P., 2009, The fragile dynamics of urban energy system transitions: The eco-cities of Graz and Freiburg in retrospect, "Cities and energy transitions: past, present, future", Autun

SALAR, 2009, Local Action on Climate Change - Swedish Experiences, Stockholm

Söderholm P., Pettersson M., Ek K., Michanek G., 2007, "Policy effectiveness and the diffusion of carbon-free energy technology : the case of wind power", in Lundgvist L. J., Biel A., From Kyoto to the Town Hall: Making International and National Climate Policy Work at the Local Level, Earthscan Publications, London, p. 93-118

Theys J., 2009, Scénarios pour une ville post-carbone, Constructif $n^{\circ} 23$, juillet 2009, http://www.constructif.fr/Article_43_78_602/Scenarios_pour_une_ville_post_carbone.html Worldwatch Institute, 2007, State of the World 2007: Our Urban Future, W. W. Norton, New York Wuppertal Institute, 2009, Sustainable Urban Infrastructure, Munich Edition - Path toward a carbonfree future, Siemens AG

\section{NOTES}

1. Définition: L'énergie grise correspond à la somme de toutes les énergies nécessaires à la production, à la fabrication, à l'utilisation et enfin au recyclage des matériaux ou des produits industriels. 


\section{RÉSUMÉS}

Dans un contexte d'urgence climatique et de pics énergétiques, la dimension locale s'avère être une échelle stratégique pour relever le défi du facteur 4 . C'est ce que nous tenterons de questionner au cœur de cet article. Nous examinerons les freins et les leviers que rencontrent les initiatives locales en Europe, en matière de politiques sectorielles, énergétiques et climatiques, ainsi que de gouvernance, en nous appuyant d'exemples précurseurs.

In a climate emergency and peak oil context, the local dimension proves to be a strategical scale to take up the challenge of factor 4 . It is what we will try to question in this article. We will examine brakes and levers which meet the local initiatives in Europe, in sectoral, energy-climate policies and governance, by leaning of forerunners examples.

\section{INDEX}

Keywords : brakes, city, energy climate plan, energy transition, governance, levers, conflict of temporalities, path dependency, territory

Mots-clés : freins, gouvernance, initiatives locales, plan climat énergie, leviers, sentiers de dépendance, territoire, transition énergétique, ville

\section{AUTEUR}

\section{ELSA MOR}

Elsa Mor, doctorante au Laboratoire Eso - Le Mans, UMR 6590 Université du Maine 